\title{
Mind your myco-robes
}

Fungi are part of the gut too, with new implications for intestinal health

The gut microbiome has emerged as a considerable variable in health and disease. Microbes can be pathogenic in their own right, but disruptions in "normal" community composition can have cascading effects that can cause or aggravate disease. Most studies in the growing microbiome literature have focused on the effects of bacteria. But bacteria aren't the exclusive colonizers of human and animal guts-fungi, viruses, and archaea also turn up, though with few considerations of their role in the microbial community or how they influence their hosts.

June Round, an immunologist at the University of Utah who studies the interactions between microbes and the immune system, has turned her attention to our fungal inhabitants, known as the mycobiota. "We know they [fungi] are very prevalent in both human and animal guts," she says, "but we know nothing about them." There are, however, clinical signs that they might not just be passive members of the gut microbiome. Anti-Saccharomyces cerevisiae antibodies, tuned to the presence of the fungi commonly known as brewer's yeast, are a diagnostic tool to distinguish Crohn's disease from ulcerative colitis. "This suggested to us that the immune response against fungi might be involved in IBD (inflammatory bowel disease), but nothing has been done to really connect those two before," she says. A new paper explores those connections (Sci. Transl. Med. 9, eaaf9044; 2017).

Round and her lab tested the immune and metabolic responses of mice that were chemically induced to model colitis after they were fed one of two common yeasts considered to be representative of major fungal phyla found in the gut: $S$. cerevisiae and another, Rhodotorula aurantiaca, that is often present in patients with IBD. The mice housed in Utah's vivarium are typically fungi-free,

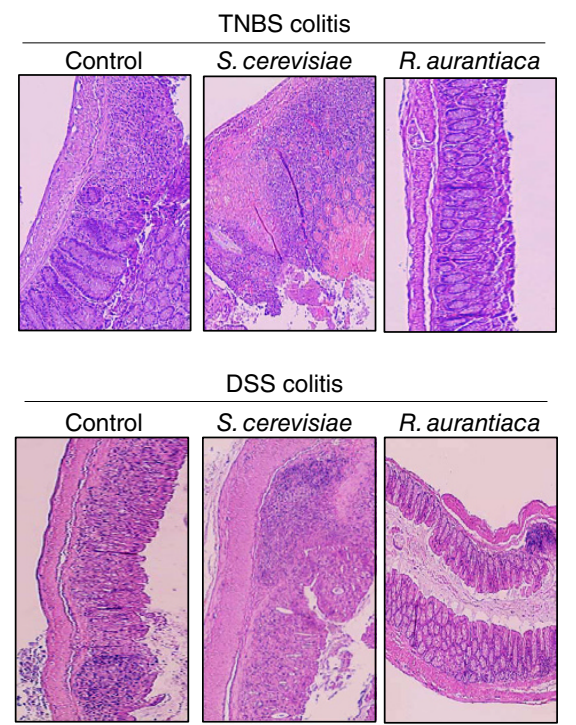

Histological changes in the intestines after colitis. Image adapted from Sci. Transl. Med. 9 , eaaf9044; 2017.

offering a de novo look at their reaction to the introduction of yeast, Round explains. The amount of yeast introduced was fairly representative of that observed normally in humans, she says, and the yeast established stable colonies prior to be induction of colitis. "It becomes part of the normal microbiota. The mice seem normal. You look at the histology of their intestines, it seems normal. So you need a trigger, this second hit to get that exacerbation of disease."

Such was the case with Saccharomyces. Mice colonized with that yeast experienced worsened symptoms of colitis, while those with Rhodotorula seemed to be neither helped nor harmed. It seems to the researchers that certain species of yeast, though they may not be the sole cause of IBD (it's a complex and multifactorial condition thought to have a genetic component), can further aggravation.

How Saccharomyces worsen colitis, however, was unexpected. The researchers observed a metabolic reaction, rather an immune response. Saccharomyces appears to hijack the metabolism of purine, which, when disrupted, causes an accumulation of uric acid. This in turn disrupts the intestinal epithelium and barrier function, causing an even leakier gut than in controls without the yeast.

That observation, however, has a translational upside: an existing drug, allopurinol. Though marketed to treat gout and kidney stones, allopurinol decreases uric acid build-up. Mice given the drug during the study had improved outcomes. Previous human studies have suggested that uric acid accumulation might be an issue for patients with IBD, but none attempted to treat that variable. Instead, most IBD treatments rely on anti-inflammatory drugs. However, if the underlying issue in certain patients is metabolic, the use of anti-inflammatories may be ineffective and unnecessarily compromise the patient's immune system. A drug like allopurinol, which has already been proven to be safe, would be a preferable alternative.

The obvious next step from this paper, Round says, is to dig deeper into the mechanism by which Saccharomyces induces the purine pathway in its hosts. But there is much to be done to improve our broader understanding of the mycobiota as well. The results of this study suggest that the presence of Saccharomyces can aggravate IBD, but any potential benefits are still unknown. She would also like to explore how different fungi interact not just with the host, but with other members of the microbiome, including bacteria.

Though bacteria may compromise the majority of the gut microbiome and attract the most attention as a result, they don't exist in isolation. Fungi and other biotas still unexplored may prove just as important in a community as complex as the gut. Ellen P. Neff 